\title{
Análise do processo de faturamento de contas de internação hospitalar combinando BPMN e Simulação
}

\author{
Rodrigo Vargas ${ }^{1}$, Thais Webber ${ }^{1}$, Ricardo Czekster $^{1}$, Rita G. Berardi ${ }^{2}$ \\ ${ }^{1}$ Departamento de Computação - Universidade de Santa Cruz do Sul (UNISC) \\ Santa Cruz do Sul - RS - Brazil \\ ${ }^{2}$ Departamento Acadêmico de Informática - Universidade Tecnológica Federal do \\ Paraná (UTFPR) - Curitiba - PR - Brazil \\ \{rodrigoro, thaiscs, ricardoc\}@unisc.br, ritaberardi@utfpr.edu.br
}

\begin{abstract}
This work focuses on an inpatient accounting process from a hospital, combining investigations in Business Process Management (BPM) and Discrete Event Simulation (DES) to support process improvement initiatives in philanthropic hospitals. For the mapping phase we have used BPMN (Business Process Modeling \& Notation) as process documentation, a database from the ERP (Enterprise Resource Planning) system belonging to the hospital and interviews with management staff. After time and resource monitoring of activities we propose DES simulations. Our preliminary results indicate bottlenecks and possibilities for process improvement.
\end{abstract}

Resumo. Este artigo foca na análise do processo de faturamento de contas hospitalares de internação, em uma investigação que combina os temas gestão de processos de negócio (BPM) e simulação de eventos discretos (DES) para apoiar iniciativas de melhorias em processos de negócio em hospitais filantrópicos. Para condução da fase de mapeamento utilizou-se a notação BPMN (Business Process Modeling \& Notation) como documentação do processo, uma base em dados do sistema ERP (Enterprise Resource Planning) hospitalar e entrevistas com gestores. Após coletas de tempos e recursos nas atividades são propostas simulações DES. Os resultados preliminares apontam gargalos e possibilidades de melhoria do processo.

\section{Introdução}

O processo de faturamento de contas hospitalares oriundas de serviços de saúde é o mais relevante, extenso e complexo conjunto de atividades administrativas em um hospital com ou sem fins lucrativos. Este processo que requer a participação de vários setores e equipes multiprofissionais é de onde são originadas quase todas as receitas operacionais do hospital [Salu 2015]. Na elaboração da conta hospitalar, quem define o que será adquirido enquanto o paciente estiver internado é prioritariamente o médico, que entre outras funções tem, muitas vezes, também o papel de restringir a necessidade e a quantidade dos recursos e insumos nos processos de assistência ao paciente. $\mathrm{O}$ custo dos itens em um hospital é atribuído via contratos e regras que definem a abrangência e escopo de cobertura dos convênios. Estes podem ser públicos, por exemplo, o SUS (Sistema Único de Saúde) ou particulares.

A visão dos processos hospitalares através de novas metodologias como BPM (Business Process Management), iniciado pelo levantamento e modelagem de 
atividades e fluxos, busca, sobretudo, entendimento das necessidades de melhorias para o negócio [Capote 2011], a citar: atendimento das necessidades e expectativas de gestão e acesso a informações, métricas e indicadores para ações estratégicas, administrativas, tomada de decisão, identificação da necessidade de reestruturação, agregando valor ao produto ou serviço.

Hoje em dia, a maioria das instituições de saúde [Salu 2015] tem o seu processo de faturamento intrínseco ao sistema de gestão integrada (ERP - Enterprise Resource Planning) implantado, o que torna possível a extração de conhecimento. Esta extração se dá através de consultas ao banco de dados e análises documentais sobre o processo e seu desempenho em tempo e utilização de recursos [Bpmcbok 2009]. Indicadores de desempenho (KPI - Key Performance Indicators) podem ser derivados desta análise dentro do contexto hospitalar, como por exemplo, tempo total de hospitalização (LoSLength of Stay), e custo médio de alta (CD - Average Cost per Discharge) que é a soma dos custos associados ao paciente (medicamentos, hotelaria, alimentação, etc.) sem considerar custos indiretos (eletricidade, manutenção predial, etc.). A simulação orientada a eventos (DES - Discrete Event Simulation) é outra abordagem que permite replicar fluxos de processos com diferentes alocações para recursos e comportamentos de entidades. Pode fornecer uma análise quantitativa na forma de KPI para busca de melhorias [Rossetti 2010], [Chwif e Medina 2010].

Este trabalho busca analisar, compreender e simular o processo de faturamento de contas de pacientes internados com objetivo de identificar melhorias no processo, em especial a redução do tempo de faturamento após a alta do paciente, um dos indicadores críticos do setor. Esta redução implica em coerência do fluxo de caixa para instituição, evitando perdas financeiras devido ao não cumprimento de prazos estabelecidos em contratos com operadoras e planos de saúde. A modelagem de processos nesta pesquisa é realizada pela aplicação de conceitos e padrões do BPM (Business Process Management) [Capote 2012] aliados ao potencial da simulação DES [Rossetti 2010] com a geração de cenários de alocação de recursos e tempos médios de atividades. São utilizados dados coletados de um ERP em produção, em uma perspectiva de análise quantitativa de sistemas por amostragem computacional [Chwif e Medina 2010].

\section{Motivação para modelagem e simulação de processos}

Dada a complexidade envolvida no processo de faturamento e as dificuldades para configuração do ERP de forma que reflita as regras de faturamento, é necessário medir previamente o impacto de alterações no sistema de gestão integrada, visando evitar situações indesejadas e modificações que comprometam o desempenho. Uma alternativa para trazer estas estimativas de resultados e comportamentos esperados, sobre mudanças sugeridas ao processo, dentro de uma abordagem científica e estatística, é o uso de técnicas de modelagem e simulação [Chwif e Medina 2010].

A simulação é uma ferramenta poderosa que é aplicada para apoiar diversas decisões de gestão, em segmentos da indústria, ciências humanas, administração, entre outros [Rossetti 2010]. Em relação à simulação computacional em saúde e cuidados de saúde, a quantidade de artigos cresceu substancialmente a partir da década de 80 [Gunal e Pidd 2010]. Gunal e Pidd (2010) constataram que DES tem sido amplamente utilizada na modelagem de sistemas em saúde, mostrando que a quantidade de artigos publicados aumentou acentuadamente a partir de 2004. Na revisão bibliográfica deste trabalho os 
autores concentraram a avaliação nos trabalhos recentes relacionados com modelagem de processos utilizando DES para cenários hospitalares com resultados satisfatórios.

Zhong et al. (2014) reforçam o apoio ao redesenho de processo com base na simulação DES utilizando o software Simula8. Foram criados modelos de simulação para analisar fluxos de pacientes de um setor hospitalar de pediatria. O objetivo foi identificar variações desnecessárias nos processos de atendimento e assim, buscar eficiência de equipes e utilização de recursos no tratamento dos pacientes. Arcanjo e Amaral (2016) realizaram o estudo em uma Unidade de Pronto Atendimento, mapeando os principais processos por onde o paciente passa até seu atendimento. Com uma amostra significativa do fluxo de pacientes foram obtidos os tempos de chegada e saída de cada paciente, em um modelo de simulação no software Arena ${ }^{1}$ [Rossetti 2010].

O uso de padrões visuais de modelagem como BPMN (Business Process Modeling \& Notation) [Bpmcbok 2009] para o entendimento do fluxo do processo se faz adequado tanto para sua tradução para modelos de simulação voltados ao estudo de comportamento de enfileiramento para quantificação de índices clássicos como vazão, tempo de espera, população e utilização [Chwif e Medina 2010], como também serve para comunicar e trocar experiências com gestores de forma facilitada e não ambígua sobre as particularidades das atividades e atores envolvidos nos processos [Capote 2011]. A simulação computacional também pode auxiliar nesta transposição de barreiras [Rossetti 2010], ou seja, diminuir a insegurança frente à necessidade de mudança e realinhamento de processos institucionais.

\section{Estudo de caso}

Foi escolhido um ambiente hospitalar do interior do Estado do Rio Grande do Sul para o desenvolvimento deste trabalho. Neste contexto, o Setor de Faturamento foi analisado, com consentimento prévio do Comitê de Ética, de forma a coletar e organizar os dados dos vários processos e atividades relacionadas à administração de convênios e planos de saúde no âmbito da saúde suplementar e dos sistemas [SUS 2006]. Dentre as atividades realizadas, citam-se algumas: cadastro de convênios, cadastro de taxas, valores e honorários, manutenção de tabelas de cobrança, configurações das formas de cobrança de cada convênio, elaboração de relatórios com dados de faturamento, faturamento de contas SUS, e faturamento de contas convênios da saúde suplementar [Salu 2015].

Para esta pesquisa é necessário abordar questões específicas sobre o ambiente de negócio dos hospitais, como ocorrem os registros dos produtos e serviços consumidos quando um paciente está em tratamento de internação. Também são necessários conhecimentos sobre as relações entre hospitais, planos de saúde e nuances que permitem qualificar o entendimento do processo de faturamento, alguns de seus problemas e seus objetivos [Salu 2015], [SUS 2006]. Neste caso, a disciplina BPM [Capote 2012] traz um novo modelo organizacional que prioriza a busca contínua de melhorias, sendo adequado para a condução do estudo de caso quanto ao levantamento e redesenho do processo. Observou-se que todas as atividades e processos hospitalares estão de alguma forma inter-relacionados, seja pelos atores envolvidos ou pela execução colaborativa e dependência das tarefas em diferentes setores. Neste estudo serão consideradas somente as atividades diretamente aplicadas ao processo de faturamento

\footnotetext{
${ }^{1}$ Disponível em https://www.arenasimulation.com/
} 
de contas de convênios de saúde suplementar, pela sua representatividade no contexto de gestão estratégica do hospital voluntário neste estudo.

O mapeamento das atividades para um modelo BPMN [Bpmcbok 2009] ocorreu através de duas abordagens: inicialmente, a top-down apresentada por Capote (2011) que visa obter uma visão do processo da perspectiva dos níveis estratégicos. Em seguida, pela perspectiva bottom-up que busca o conhecimento do processo pela visão da execução do fluxo de suas atividades. Abordar o processo destas maneiras foi importante para o entendimento detalhado das atividades e para inferir o grau de maturidade do processo. Utilizaram-se técnicas como: contato formal, por correio eletrônico; entrevistas semiestruturadas com a coordenação do Setor de Faturamento; acompanhamento das rotinas de trabalho, e consultas ao ERP [Bpmcbok 2009].

A modelagem de simulação (DES) tem como objetivo projetar mudanças que possam contribuir para identificação de gargalos a partir da descrição do fluxo e dos comportamentos que seguem distribuições de probabilidades, cujas equações sofrem um ajuste aplicado a partir dos dados coletados no ERP e modelados por técnicas estatísticas [Rossetti 2010]. Objetiva-se utilizar as simulações como argumentos quantitativos para as propostas de mudanças e redesenhos dos processos institucionais. A tradução do modelo BPMN para um modelo de simulação, neste trabalho, para o ambiente do software Arena, foi uma segunda etapa do levantamento de dados do ERP, estes avaliados quanto à dispersão e presença de outliers antes da geração das equações de comportamento de cada atividade e entidades do modelo [Chwif e Medina 2010].

\section{Resultados preliminares}

Com base nas entrevistas e observações foi possível elaborar a documentação do processo As Is - como é [Bpmcbok 2009]. A ferramenta de modelagem utilizada foi a Bizagi Modeler ${ }^{2}$. A validação do modelo de processo de faturamento foi feita com gestores do setor com experiência no faturamento de contas de convênios particulares e conhecimento da notação BPMN devido a intervenções anteriores em outros setores para o desenvolvimento organizacional. Apontaram que o modelo está suficientemente genérico, logo pode ser aplicado a todos os convênios particulares. Houve concordância quanto à sequencialidade e paralelização de atividades, sendo as anotações no diagrama quanto aos volumes de recursos apontados como corretos. Os itens pontuados foram baseados nas recomendações constantes no Bpmcbok (2009) e autor Capote (2011) para finalizar a validação do diagrama. Os próximos passos incluem a identificação de indicadores de desempenho (KPI) do processo de faturamento, refinamento do modelo no software Arena, validação da tradução realizada do fluxo de atividades do processo com as equações geradas pela análise estatística dos dados do ERP. A partir do cenário As $I s$ de simulação projetar as mudanças para descoberta de oportunidades de melhorias do processo de faturamento.

Os cenários identificados como melhorias, ainda na etapa de modelagem, foram preliminarmente simulados conforme modelo $A s$ Is. A retirada de atividades desnecessárias, por exemplo, na atividade de controle de prontuários, que atualmente passa por outros setores e atores antes da atribuição final ao faturamento, enquanto poderia ser entregue diretamente para equipe de faturistas, que poderia efetuar a

\footnotetext{
${ }^{2}$ Disponível em http://www.bizagi.com/pt/produtos/bpm-suite/modeler
} 
certificação da entrega via sistema ERP, pois existe um módulo de controle de prontuários. Através de simulações preliminares foi possível perceber uma redução de $15 \%$, cerca de três (3) dias dos 21 dias no tempo de faturamento, ao reorganizar estas atribuições de tarefas. A Tabela 1 mostra um exemplo do conjunto de dados de entrada para a simulação que foi coletado para parametrização no software Arena.

Tabela 1. Exemplos de dados de entrada coletados para a simulação

\begin{tabular}{clll}
\hline Variável & Descrição & Unidade & Disponível \\
\hline A & Intervalo entre chegadas & segundos & ERP \\
B & Tempo de tratamento & horas & ERP \\
C & Intervalo entre altas & segundos & ERP \\
D & Tempo para envio ao faturamento & horas & Regra Interna \\
E & Tempo para protocolar entrega & segundos & Entrevista \\
F & Tempo envio para faturista & minutos & Entrevista \\
G & Tempo controle contas em aberto & dias & Entrevista \\
H & Valor da conta & R\$ & ERP \\
I & Tipo convênio & inteiro & ERP \\
J & Tempo conferência & minutos & Entrevista \\
K & Tempo resolução pendência & minutos & Entrevista \\
M & Tempo para realizar lançamentos & minutos & ERP \\
N & Quantidade de contas & inteiro & ERP \\
P & Tempo para auditoria & minutos & Entrevista \\
Q & Tempo Total do Processo & dias & ERP \\
\hline
\end{tabular}

Fonte: Elaborado pelo autor.

Outro cenário é estabelecer um prazo menor e mais homogêneo para as atividades de ajuste de contas, que passariam a ser realizadas em até 24 (vinte e quatro) horas após a alta do paciente. Como resultado disso, a simulação demonstrou que o prazo de faturamento seria reduzido, em média, cerca de um (1) dia - conforme simulações realizadas com dados e probabilidades oriundas do sistema ERP.

\section{Conclusão}

Apesar de motivadores os resultados preliminares de simulações, pode-se ressaltar que a simulação DES é bastante sensível à entrada de dados, ou seja, se as informações e dados de entrada e os tempos médios para realização das atividades do processo forem precisos, a simulação fornecerá resultados confiáveis. Portanto, considerando um ambiente dinâmico como o hospitalar, dependendo do problema que se pretende simular, é necessário um longo período de observação e interação com os atores do processo para que sejam gerados parâmetros com a qualidade necessária para simulações complexas. Um exemplo da sensibilidade é o fato de que por não haverem dados de auditoria das alterações das contas por um período superior a 60 (sessenta) dias foi necessário considerar este limite de tempo de simulação, pois com tempos superiores, os resultados da simulação perdem precisão em relação ao cenário real.

Com base nos resultados apurados durante a pesquisa é possível concluir que a simulação DES pode ser aplicada no escopo da análise de processos hospitalares como uma ferramenta auxiliar de análise para melhoria de processos de faturamento. Deve ser complementada por ferramentas que possibilitem uma melhor coleta de dados uma vez que, a apesar da grande quantidade de atividades realizada via ERP, onde é possível 
obter dados suficientemente precisos para uma simulação completa. Há atividades onde não existem quaisquer registros e que são suscetíveis a interpretações do analista, que nem sempre tem as ferramentas e a experiência necessária para uma boa estimativa.

\section{Agradecimentos}

Os autores Dr. Ricardo M. Czekster e Dra. Thais Webber agradecem o apoio dado pelo CNPq (processo n.403294/2016-9), projeto que envolve análise de dados para melhorar a eficiência de processos. Os autores também agradecem a colaboração dos gestores e profissionais de saúde do Hospital Santa Cruz (HSC).

\section{Referências}

Bpmcbok (2009), "Guia para o Gerenciamento de Processos de Negócio - Corpo Comum de Conhecimento", BPM CBOK V.3.0, 1.ed., Brasil, 2013. $<$ www.abpmp.org/resource/resmgr/Docs/ABPMP_CBOK_Guide_Portuguese.pdf $>$. Acesso em: 5 março. 2018.

Arcanjo, C.F.D.; Amaral, T.M. (2016), "Mapeamento de fluxo de pacientes e simulação de eventos discretos no sistema público de saúde: Um caso prático em uma unidade de pronto atendimento em Juazeiro-BA", In: XXXV Encontro Nacional de Engenharia de Produção, N.13.

Capote, G. (2011), “Guia para formação de analistas de processos: business process management”, 1. ed., Rio de Janeiro, RJ: ISBN 978-14-563-660-18.

Capote, G. (2012), "BPM para todos: uma visão geral abrangente, objetiva e esclarecedora sobre gerenciamento de processos de negócio", BPM, 1. ed., Rio de Janeiro, RJ: ISBN 978-1470005-41-2.

Chwif, L.; Medina, A.C. (2010), "Modelagem e simulação de eventos discretos: teoria \& aplicações”, 3. ed., São Paulo, SP: Ed. dos Autores, 2010. ISBN 978-85-905978$3-4$.

Gunal, M.; Pidd, M. (2010), "Discrete event simulation for performance modelling in healthcare: a review of the literature", Journal of Simulation, Palgrave Macmillan Ltd., V. 4, N. 1, P. 42-51, ISSN 1747-7778.

Rossetti, M.D. (2010), "Simulation modeling and Arena", New Jersey, USA: John Wiley \& Sons, 2010. ISBN 978-0-470-09726-7.

Salu, E.J. (2015), "Modelo GFACH Gestão em Faturamento, Auditoria de Contas e Administração Comercial Hospitalar”, 1. ed., São Paulo, SP: Edição do Autor, 2015. ISBN 978-85-917645-5-6.

SUS. Ministério da Saúde. (2006), "Entendendo o SUS”, Brasília, DF, 2006. $<$ www.portalarquivos.saude.gov.br/images/pdf/2013/agosto/28/cartilha-entendendoo-sus-2007.pdf>. Acesso em: 5 março. 2018.

Zhong, X. et al. (2014), "Primary care redesign: A simulation study at a pediatric clinic". In: 2014, IEEE International Conference on Automation Science and Engineering (CASE), P. 546-551. ISSN 2161-8070. 